Georgian Mathematical Journal

Volume 11 (2004), Number 1, 99-104

\title{
INTEGRABILITY AND $L^{1}$-CONVERGENCE OF MODIFIED SINE SUMS
}

\author{
KULWINDER KAUR, S. S. BHATIA, AND BABU RAM
}

\begin{abstract}
New modified sine sums are introduced and a criterion for the $L^{1}$-convergence of these modified sine sums under a new class $K$ is obtained. Also a necessary and sufficient condition for the $L^{1}$-convergence of the cosine series is deduced as a corollary.
\end{abstract}

2000 Mathematics Subject Classification: 42A20, 42A32.

Key words and phrases: $L^{1}$-convergence, modified sine sums, conjugate Fejer kernel.

\section{INTRODUCTION}

Consider the cosine series

$$
g(x)=\frac{a_{0}}{2}+\sum_{k=1}^{\infty} a_{k} \cos k x
$$

with partial sums defined by $S_{n}(x)=\frac{a_{0}}{2}+\sum_{k=1}^{n} a_{k} \cos k x$ and let

$$
g(x)=\lim _{n \rightarrow \infty} S_{n}(x) .
$$

Concerning the $L^{1}$-convergence of cosine series (1.1) Kolmogorov [2] proved the following theorem:

Theorem A. If $\left\{a_{n}\right\}$ is a quasi-convex null sequence, then for the $L^{1}$ convergence of the cosine series (1.1) it is necessary and sufficient that $\lim _{n \rightarrow \infty} a_{n} \log n=0$.

The case of this theorem in which the sequence $\left\{a_{n}\right\}$ is convex, was established by Young [7]. That is why this Theorem A is sometimes known as YoungKolmogorov Theorem.

Rees and Stanojević [5] have introduced modified cosine sums

$$
g_{n}(x)=\frac{1}{2} \sum_{k=0}^{n} \triangle a_{k}+\sum_{k=1}^{n} \sum_{j=k}^{n}\left(\triangle a_{j}\right) \cos k x .
$$

Garret and Stanojević [1], Ram [4] and Singh and Sharma [6] studied the $L^{1}$-convergence of these cosine sums under different sets of conditions on the coefficients $a_{n}$. 
Later on, Kumari and Ram [3], introduced new modified cosine and sine sums as

$$
f_{n}(x)=\frac{a_{0}}{2}+\sum_{k=1}^{n} \sum_{j=k}^{n} \triangle\left(\frac{a_{j}}{j}\right) k \cos k x
$$

and

$$
g_{n}(x)=\sum_{k=1}^{n} \sum_{j=k}^{n} \triangle\left(\frac{a_{j}}{j}\right) k \sin k x
$$

and have studied their $L^{1}$ - convergence under the condition that the coefficients $a_{n}$ belong to different classes of sequences. Also, they deduced some results about the $L^{1}$-convergence of cosine and sine series as corollaries.

We introduce here new modified sine sums as

$$
K_{n}(x)=\frac{1}{2 \sin x} \sum_{k=1}^{n} \sum_{j=k}^{n}\left(\triangle a_{j-1}-\triangle a_{j+1}\right) \sin k x .
$$

The aim of this paper is to study the $L^{1}$-convergence of these modified sine sums $K_{n}(x)$ and to obtain an analogue of Theorem A of Kolmogorov for a newly defined class $\mathbf{K}$ of coefficient sequences defined as follows:

Definition. If $a_{k}=o(1), k \rightarrow \infty$, and

$$
\sum_{k=1}^{\infty} k\left|\triangle^{2} a_{k-1}-\triangle^{2} a_{k+1}\right|<\infty \quad\left(a_{0}=0\right),
$$

then we say that $\left\{a_{k}\right\}$ belongs to the class $\mathbf{K}$.

\section{Main Result}

The main result is the following theorem:

Theorem 1. Let the sequence $\left\{a_{n}\right\}$ belong to the class $\mathbf{K}$, then $K_{n}(x)$ converges to $g(x)$ in $L^{1}$-norm.

Proof. We have

$$
\begin{aligned}
S_{n}(x) & =\frac{a_{0}}{2}+\sum_{k=1}^{n} a_{k} \cos k x=\frac{1}{2 \sin x} \sum_{k=1}^{n} a_{k} \cos k x 2 \sin x \\
& =\frac{1}{2 \sin x} \sum_{k=1}^{n} a_{k}[\sin (k+1) x-\sin (k-1) x] \\
& =\frac{1}{2 \sin x} \sum_{k=1}^{n}\left(a_{k-1}-a_{k+1}\right) \sin k x+a_{n+1} \frac{\sin n x}{2 \sin x}+a_{n} \frac{\sin (n+1) x}{2 \sin x} .
\end{aligned}
$$


Applying Abel's transformation, we have

$$
\begin{aligned}
S_{n}(x)= & \frac{1}{2 \sin x}\left(\sum_{k=1}^{n}\left(\triangle a_{k-1}-\triangle a_{k+1}\right) \tilde{D}_{k}(x)+\left(a_{n}-a_{n+2}\right) \tilde{D}_{n}(x)\right) \\
& +a_{n+1} \frac{\sin n x}{2 \sin x}+a_{n} \frac{\sin (n+1) x}{2 \sin x}
\end{aligned}
$$

where $\tilde{D}_{k}(x)$ denotes Dirichlet conjugate kernel. Thus

$$
g(x)=\lim _{n \rightarrow \infty} S_{n}(x)=\frac{1}{2 \sin x} \sum_{k=1}^{\infty}\left(\triangle a_{k-1}-\triangle a_{k+1}\right) \tilde{D}_{k}(x),
$$

if the series is convergent. Also,

$$
\begin{aligned}
K_{n}(x) & =\frac{1}{2 \sin x} \sum_{k=1}^{n} \sum_{j=k}^{n}\left(\triangle a_{j-1}-\triangle a_{j+1}\right) \sin k x \\
& =\frac{1}{2 \sin x}\left(\sum_{k=1}^{n}\left(a_{k-1}-a_{k+1}\right) \sin k x-\left(a_{n}-a_{n+2}\right) \tilde{D}_{n}(x)\right) .
\end{aligned}
$$

Applying Abel's transformation, we have

$$
K_{n}(x)=\frac{1}{2 \sin x} \sum_{k=1}^{n}\left(\triangle a_{k-1}-\triangle a_{k+1}\right) \tilde{D}_{k}(x) .
$$

Since $\left|\frac{\tilde{D}_{k}(x)}{2 \sin x}\right|=O(k)$ and

$$
\sum_{k=1}^{\infty} k\left|\triangle^{2} a_{k-1}-\triangle^{2} a_{k+1}\right|<\infty
$$

the series

$$
\frac{1}{2 \sin x} \sum_{k=1}^{\infty}\left(\triangle a_{k-1}-\triangle a_{k+1}\right) \tilde{D}_{k}(x)
$$

converges.

Hence $\lim _{n \rightarrow \infty} K_{n}(x)=g(x)$ exists.

Thus

$$
\begin{aligned}
g(x)-K_{n}(x) & =\frac{1}{2 \sin x} \sum_{k=n+1}^{\infty}\left(\triangle a_{k-1}-\triangle a_{k+1}\right) \tilde{D}_{k}(x) \\
& =\lim _{m \rightarrow \infty}\left(\frac{1}{2 \sin x} \sum_{k=n+1}^{m}\left(\triangle a_{k-1}-\triangle a_{k+1}\right) \tilde{D}_{k}(x)\right) .
\end{aligned}
$$

The use of Abel's transformation gives

$$
g(x)-K_{n}(x)=\frac{1}{2 \sin x} \lim _{m \rightarrow \infty}\left[\sum_{k=n+1}^{m-1}(k+1)\left(\triangle^{2} a_{k-1}-\triangle^{2} a_{k+1}\right) \tilde{F}_{k}(x)\right.
$$




$$
\begin{aligned}
& \left.+(m+1)\left(\triangle a_{m-1}-\triangle a_{m+1}\right) \tilde{F}_{m}(x)-(n+1)\left(\triangle a_{n}-\triangle a_{n+2}\right) \tilde{F}_{n}(x)\right] \\
= & \frac{1}{2 \sin x}\left[\sum_{k=n+1}^{\infty}(k+1)\left(\triangle^{2} a_{k-1}-\triangle^{2} a_{k+1}\right) \tilde{F}_{k}(x)\right. \\
& \left.-(n+1)\left(\triangle a_{n}-\triangle a_{n+2}\right) \tilde{F}_{n}(x)\right]
\end{aligned}
$$

where

$$
\tilde{F}_{k}(x)=\frac{1}{k+1} \sum_{j=0}^{k} \tilde{D}_{j}(x)
$$

denotes the conjugate Fejer kernel. Now

$$
\begin{aligned}
\int_{-\pi}^{\pi} \mid g(x)- & K_{n}(x) \mid d x \\
= & \int_{-\pi}^{\pi} \mid \frac{1}{2 \sin x}\left[\sum_{k=n+1}^{\infty}(k+1)\left(\triangle^{2} a_{k-1}-\triangle^{2} a_{k+1}\right) \tilde{F}_{k}(x)\right. \\
& -(n+1)\left(\triangle a_{n}-\triangle a_{n+2}\right) \tilde{F}_{n}(x) d x \mid \\
\leq & C\left[\sum_{k=n+1}^{\infty}(k+1)\left|\left(\triangle^{2} a_{k-1}-\triangle^{2} a_{k+1}\right)\right| \int_{-\pi}^{\pi}\left|\tilde{F}_{k}(x)\right| d x\right. \\
& \left.+(n+1)\left|\left(\triangle a_{n}-\triangle_{n+2}\right)\right| \int_{-\pi}^{\pi}\left|\tilde{F}_{n}(x)\right| d x\right] .
\end{aligned}
$$

But $\frac{1}{\pi} \int_{-\pi}^{\pi}\left|\tilde{F}_{k}(x)\right| d x=1$, and

$$
\begin{aligned}
\mid\left(\triangle a_{n}\right. & \left.-\triangle a_{n+2}\right)|=| \sum_{k=n}^{\infty}\left(\triangle^{2} a_{k}-\triangle^{2} a_{k+2}\right) \mid \\
& =\left|\sum_{k=n+1}^{\infty} \frac{k}{k}\left(\triangle^{2} a_{k-1}-\triangle^{2} a_{k+1}\right)\right| \\
& \leq \frac{1}{n+1}\left|\sum_{k=n+1}^{\infty} k\left(\triangle^{2} a_{k-1}-\triangle^{2} a_{k+1}\right)\right|=o\left(\frac{1}{n+1}\right) .
\end{aligned}
$$

Thus we have

$$
\int_{-\pi}^{\pi}\left|g(x)-K_{n}(x)\right| d x=O\left(\sum_{k=n+1}^{\infty}(k+1)\left|\left(\triangle^{2} a_{k-1}-\triangle^{2} a_{k+1}\right)\right|\right)
$$




$$
+o(1)=o(1)
$$

by $(1.3)$.

Corollary. If $\left\{a_{n}\right\}$ belongs to the class $\boldsymbol{K}$, then the necessary and sufficient condition for the $L^{1}$-convergence of the cosine series (1.1) is $\lim _{n \rightarrow \infty} a_{n} \log n=0$.

Proof. We have

$$
\begin{aligned}
\| S_{n}(x) & -g(x)\|\leq\| S_{n}(x)-K_{n}(x)\|+\| K_{n}(x)-g(x)\|=\| K_{n}(x)-g(x) \| \\
& +\left\|\left(a_{n}-a_{n+2}\right) \frac{\tilde{D}_{n}(x)}{2 \sin x}+a_{n+1} \frac{\sin n x}{2 \sin x}+a_{n} \frac{\sin (n+1) x}{2 \sin x}\right\| .
\end{aligned}
$$

Also,

$$
\begin{aligned}
\|\left(a_{n}\right. & \left.-a_{n+2}\right) \frac{\tilde{D}_{n}(x)}{2 \sin x}+a_{n+1} \frac{\sin n x}{2 \sin x}+a_{n} \frac{\sin (n+1) x}{2 \sin x} \| \\
& =\left\|K_{n}(x)-S_{n}(x)\right\| \leq\left\|K_{n}(x)-g(x)\right\|+\left\|S_{n}(x)-g(x)\right\|,
\end{aligned}
$$

and

$$
\begin{aligned}
& \left|\left(a_{n}-a_{n+2}\right)\right|=\left|\sum_{k=n}^{\infty}\left(\triangle a_{k}-\triangle a_{k+2}\right)\right| \\
& \quad=\left|\sum_{k=n+1}^{\infty} \frac{k}{k}\left(\triangle a_{k-1}-\triangle a_{k+2}-\triangle a_{k+1}+\triangle a_{k+2}\right)\right| \\
& \quad \leq \frac{1}{n+1}\left|\sum_{k=n+1}^{\infty} k\left(\triangle^{2} a_{k-1}-\triangle^{2} a_{k+1}\right)\right|=o\left(\frac{1}{n}\right) .
\end{aligned}
$$

Since $\int_{-\pi}^{\pi} \frac{\tilde{D}_{n}(x)}{2 \sin x} d x=O(n)$, we obtain

$$
\left(a_{n}-a_{n+2}\right) \int_{-\pi}^{\pi} \frac{\tilde{D}_{n}(x)}{2 \sin x} d x=O\left(\left(a_{n}-a_{n+2}\right) n\right)=o(1)
$$

Moreover,

$$
\begin{aligned}
& \int_{-\pi}^{\pi}\left|a_{n+1} \frac{\sin n x}{2 \sin x}+a_{n} \frac{\sin (n+1) x}{2 \sin x}\right| d x \\
& \leq \int_{-\pi}^{\pi} a_{n}\left|\frac{\sin n x}{2 \sin x}+\frac{\sin (n+1) x}{2 \sin x}\right| d x=a_{n} \int_{-\pi}^{\pi}\left|D_{n}(x)\right| d x \sim\left(a_{n} \log n\right) .
\end{aligned}
$$

Since $\left\|K_{n}(x)-g(x)\right\|=o(1)(n \rightarrow \infty)$, by Theorem 2.1 . 
Therefore

$$
\lim _{n \rightarrow \infty} \int_{-\pi}^{\pi}\left|g(x)-S_{n}(x)\right| d x=o(1)
$$

if and only if $\lim _{n \rightarrow \infty} a_{n} \log n=0$.

\section{REFERENCES}

1. J. W. Garrett and Č. V. Stanojević, On $L^{1}$-convergence of certain cosine sums. Proc. Amer. Math. Soc. 54(1976), 101-105.

2. A. N. Kolmogorov, Sur l'ordre de grandeur des coefficients de la series de FourierLebesgue. Bull. Polon. Sci. Ser. Sci. Math. Astronom. Phys. 1923, 73-76.

3. Suresh Kumari and BABU RAm, $L^{1}$-convergence modified cosine sum. Indian J. pure appl. Math. 19(1977), No. 11, 1101-1104.

4. Babu Ram, Convergence of certain cosine sums in the metric space L. Proc. Amer. Math. Soc. 66(1977), 257-260.

5. C. S. Rees and Č. V. Stanojević, Necessary and sufficient condition for intergrability of certain cosine sums. J. Math. Anal. Appl. 43(1973), 579-586.

6. N. Singh and K. M. Sharma, Convergence of certain cosine sums in the metric space $L$. Proc. Amer. Math. Soc. 75(1977), 117-120.

7. W. H. Young, On the Fourier series of bounded functions. Proc. London Math. Soc. 12(1913), No. 2, 41-70.

8. A. Zygmund, Trigonometric series. I. Cambridge University Press, Cambridge, 1959.

(Received 1.05.2003)

Authors' addresses:

Kulwinder Kaur and S. S. Bhatia

School of Mathematics \& Computer Applications, Thapar Institute of Engineering and Technology (Deemed University)

P. B. No. 32, Patiala (Pb.)-147004

India

E-mail address: mathkk@hotmail.com

Babu Ram

Departments of Mathematics

Maharshi Dyanand University

Rohtak, India 